2011-01-01

\title{
Proposal for a Simple Integrated Optical Ion Exchange Waveguide Polarizer with a Liquid Crystal Overlay
}

\author{
Pengfei Wang \\ Technological University Dublin, pengfei.wang@tudublin.ie \\ Yuliya Semenova \\ Technological University Dublin, yuliya.semenova@tudublin.ie \\ Jie Zheng \\ Jilin University \\ See next page for additional authors \\ Follow this and additional works at: https://arrow.tudublin.ie/engscheceart \\ Part of the Engineering Commons
}

\section{Recommended Citation \\ Wang, P. et al. (2011) Proposal for a Simple Integrated Optical Ion Exchange Waveguide Polarizer with a Liquid Crystal Overlay, Optics Communications, Vol.284, pp.979-984. doi:10.1016/j.optcom.2010.10.034}

This Article is brought to you for free and open access by the School of Electrical and Electronic Engineering at ARROW@TU Dublin. It has been accepted for inclusion in Articles by an authorized administrator of ARROW@TU Dublin. For more information, please contact arrow.admin@tudublin.ie, aisling.coyne@tudublin.ie, gerard.connolly@tudublin.ie.

Funder: Irish Research Council for Science, Engineering and Technology, co-funded by the Marie-Curie Actions under FP7. The research was partially supported by the National Natural Science Foundation of China (No. 60777038), the China-Ireland Science and Technology Collaboration Research Fund and the International cooperation project (No.20070708-3) of Jilin Provincial Science \& Technology Department of China

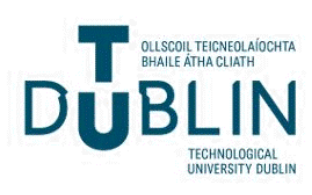




\section{Authors}

Pengfei Wang, Yuliya Semenova, Jie Zheng, Qiang Wu, Agus Hatta, and Gerald Farrell 


\title{
Proposal for a Simple Integrated Optical Ion Exchange Waveguide Polarizer with a Liquid Crystal Overlay
}

\author{
Pengfei Wang ${ }^{1,{ }^{*}}$ Yuliya Semenova ${ }^{1}$, Jie Zheng ${ }^{2}$, Qiang Wu ${ }^{1}$, Agus Muhamad Hatta ${ }^{1}$ \\ and Gerald Farrell ${ }^{1}$ \\ ${ }^{1}$ Photonics Research Center, School of Electronic and Communications Engineering, \\ Dublin Institute of Technology, Kevin Street, Dublin 8, Ireland; \\ ${ }^{2}$ State Key Laboratory of Integrated Optoelectronics, College of Electronic Science \\ and Engineering, Jilin University, Changchun 130012, P. R. China \\ *Corresponding email: pengfei.wang@dit.ie
}

\begin{abstract}
A simple, compact electrooptic polarizer based on an ion-exchanged glass channel waveguide covered with a nematic liquid crystal (LC) is proposed. A full vectorial beam propagation method is employed to simulate this device for the first time. For the cases of zero and strong LC surface anchoring, the performance of the proposed polarizer under different applied voltages is analyzed numerically. Analysis indicates that surface anchoring of the liquid crystal is a key issue influencing the performance for the proposed optical polarizer device.
\end{abstract}

Key words: Optical polarizer, glass waveguide, ion exchange, liquid crystal

\section{Introduction}

Numerous photonic integrated circuits (PICs), such as planar lightwave circuits and photonic crystal circuits, have been emerging driven by the development of optical communications and optical sensing. They have superior characteristics, such as compact size and high scalability by comparison to bulk optical devices.

Liquid crystal (LC) materials have also been widely used in photonics due to their high birefringence, superior electro-optical properties and a relatively fast response time in the order of milliseconds. Recently several authors have reported the 
use of liquid crystals combined with PICs to fabricate various photonic devices, e.g., integrated optical switches, polarization splitters, modulators, tunable filter etc [1-7].

For bulk liquid crystal based optical devices, a range of accurate models for the simulation of light behaviour have been developed in recent years. For example, matrix methods, such as the Jones matrix method or Berremann matrix method are popularly employed for theoretical prediction. When the device size is comparable to the operating wavelength, other numerical methods, such as finite-difference time domain method (FDTD) or beam propagation method (BPM) are essential to simulate the light behaviour within a PIC device that combines a LC material with an isotropic dielectric structure. In this paper, theoretical modelling and numerical simulations of integrated liquid crystal devices based on an ion-exchanged waveguide are carried out using a full-vectorial finite-difference beam propagation method (FD-BPM). Based on this for the first time an integrated compact electro-optic switchable polarizer is presented.

Ion-exchanged glass waveguide based PICs have been extensively investigated due to the numerous advantages they offer, such as very low propagation loss, low birefringence, low coupling loss with singlemode optical fibres and a relatively simple fabrication technique. A number of configurations for integrated ion exchanged glass waveguide based PICs have been proposed [8, 9]. However to the best of our knowledge, no investigations on an ion exchange waveguide combined with LC have been reported. In this paper an integrated LC-ion exchanged polarizer is proposed, designed and simulated. The proposed optical polarizer consists of an ion exchanged channel waveguide substrate covered with a LC layer. The application of an electric field above the threshold value in a direction perpendicular to the LC layer with a positive dielectric anisotropy causes changes in the LC molecular orientation from a planar alignment at the surface of the waveguide to a homeotropic alignment (perpendicular to the surface of the ion-exchanged waveguide). This results in polarisation-dependent changes of the light propagation along the ion-exchanged channel waveguide. In our simulations strong anchoring of the LC molecules to the surface of the ion-exchanged waveguide is assumed. The light propagation in the integrated device is simulated and characterized in the absence of electric field and under the influence of different applied voltages. The results indicate that surface 
anchoring conditions for the liquid crystal at the surface of the ion-exchanged waveguide are a key issue in realizing the expected function.

\section{Proposed configuration of the device and theoretical modelling tools}

\section{Proposed device configuration}

Schematic structure of the proposed device is presented in Fig. 1. The liquid crystal layer is sandwiched between the surface of the ion-exchanged glass waveguide with a deposited ITO electrode and a glass plate with a second ITO electrode. To ensure the LC directors at the surface layer are homogenously aligned in a specific direction, two polyimide alignment layers with a thickness of $100 \mathrm{~nm}$ are added between the LC and ITO layers. In the models, the refractive index of polyimide BPADA/FDA (1.672@1550nm) is chosen as an example. The whole device can be regarded as a liquid crystal cell with the planar lightwave circuit as a substrate. In simulations two possible liquid crystal molecular orientations are considered: planar alignment along the $x$-axis and a homeotropic alignment along the $y$-axis.

The ordinary and extraordinary refractive indices of the liquid crystal are $n_{o}$ and $n_{e}$, respectively. Here the nematic LC E7 is chosen with the ordinary and extraordinary refractive indices of 1.5 and 1.7 at the wavelength of $1550 \mathrm{~nm}$. The value of the refractive index for the ion exchanged waveguide $n_{c o}$ is chosen between $n_{o}$ and $n_{e}$ in the present paper. The refractive index of the glass substrate is assumed to be 1.507 and the maximum difference between the waveguide and the substrate refractive indices is chosen as 0.049 . The width of the photomask for the ion exchange is $3 \mu \mathrm{m}$.

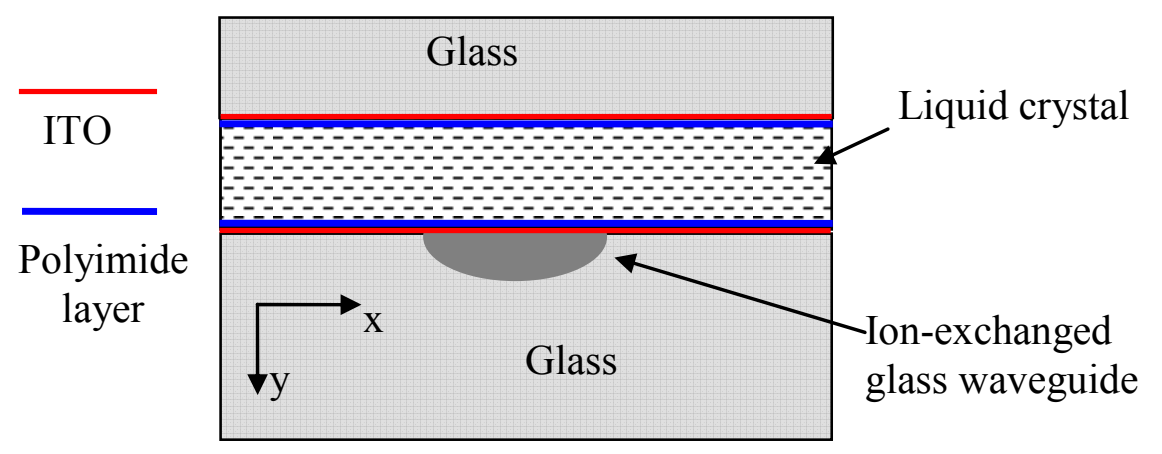


(a) TM-passing polarizer

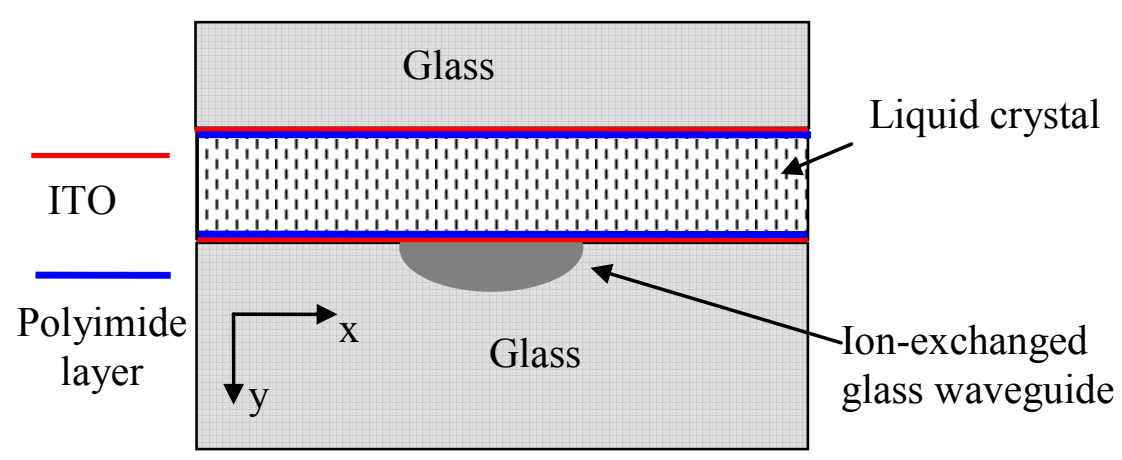

(b) TE-passing polarizer

Fig. 1. Schematic configuration (cross section) of an ion-exchanged glass waveguide covered by a layer of nematic LC as the proposed polarizer; LC molecules are along the $\mathrm{x}$-axis in configuration (a) and along the y-axis for configuration (b).

The operating principle of this optical polarizer is based on the fact that due to the birefringence of the liquid crystal, the TE and TM modes of the waveguide have different index profiles in the liquid crystal area adjacent to the waveguide and consequently have different propagation characteristics. In configuration (a) in Fig. 1, the TE mode (the polarization direction is defined in the $\mathrm{x}-\mathrm{z}$ plane) should be confined within the LC layer but the TM mode (the polarization direction is in the $y-z$ direction) propagates in both the waveguide and LC areas due to the presence of strong guiding. In configuration (b) which can be achieved by applying a threshold field to the LC layer, the TM mode will be confined within the LC layer and TE mode will propagate in both the waveguide and the LC layer near the surface of the waveguide.

To carry out the theoretical design and numerical analysis, a supermode solution for an ion-exchanged waveguide combined with a birefringent material and corresponding full-vector beam propagation method are briefly described below.

\section{Theoretical modelling tools}

The wave equation for a birefringent dielectric structure is

$$
\nabla \times \nabla \times \vec{E}-k^{2} \hat{\varepsilon}(x, y, z) \vec{E}=0
$$


where $k=2 \pi / \lambda_{0}$ and $\lambda_{0}$ is the wavelength in free-space. In the liquid crystal area, $\hat{\varepsilon}$ is the optical tensor of liquid crystals. For configuration (a): $\varepsilon_{x x}=n_{e}^{2}, \varepsilon_{y y}=\varepsilon_{z z}=n_{o}^{2}$, $\varepsilon_{m n}=0$ (where $m, n=x, y, z$, and $m \neq n$ ) and for configuration (b): $\varepsilon_{y y}=n_{e}^{2}$, $\varepsilon_{x x}=\varepsilon_{z z}=n_{o}^{2}, \varepsilon_{m n}=0$ (where $m, n=x, y, z$, and $m \neq n$ ). For both configurations, in the core area: $\varepsilon_{x x}=\varepsilon_{y y}=\varepsilon_{z z}$ is adequate to define the refractive index profile of the ionexchanged waveguide, also $\varepsilon_{m n}=0$ and in the surrounding substrate region, $\varepsilon_{x x}=\varepsilon_{y y}=\varepsilon_{z z}=n_{s u b}^{2}, \varepsilon_{m n}=0$. The x-and y-components of the electric field satisfy

$$
-\frac{\partial^{2}}{\partial z^{2}}\left[\begin{array}{l}
E_{x} \\
E_{y}
\end{array}\right]=\left[\begin{array}{cc}
P_{x x} & P_{x y} \\
P_{y x} & P_{y y}
\end{array}\right]\left[\begin{array}{l}
E_{x} \\
E_{y}
\end{array}\right]
$$

where $P_{x x} E_{x}=\frac{\partial}{\partial x}\left[\frac{1}{\varepsilon_{z z}} \frac{\partial}{\partial x}\left(\varepsilon_{x x} E_{x}\right)\right]+\frac{\partial^{2} E_{x}}{\partial y^{2}}+k^{2}\left[\left(\varepsilon_{x x}-n_{0}^{2}\right) E_{x}\right]$,

$$
\begin{aligned}
& P_{x y} E_{y}=\frac{\partial}{\partial x}\left[-\frac{\partial E_{y}}{\partial y}+\frac{1}{\varepsilon_{z z}} \frac{\partial}{\partial y}\left(\varepsilon_{y y} E_{y}\right)\right], \\
& P_{y x} E_{x}=\frac{\partial}{\partial y}\left[-\frac{\partial E_{x}}{\partial x}+\frac{1}{\varepsilon_{z z}} \frac{\partial}{\partial x}\left(\varepsilon_{x x} E_{x}\right)\right], \\
& P_{y y} E_{y}=\frac{\partial^{2} E_{y}}{\partial x^{2}}+\frac{\partial}{\partial y}\left[\frac{1}{\varepsilon_{z z}} \frac{\partial}{\partial y}\left(\varepsilon_{y y} E_{y}\right)\right]+k^{2}\left[\left(\varepsilon_{y y}-n_{0}^{2}\right) E_{y}\right],
\end{aligned}
$$

Based on these BPM equations, modeling of the beam behaviours for two polarization states in the ion exchanged waveguide covered by a liquid crystal are presented in the following section.

Modelling of the ion exchange distribution is also needed. The nonlinear diffusion equation for ion-exchange is [9-13]

$$
\frac{\partial}{\partial t} C=\frac{D_{A}}{1-\alpha C}\left[\nabla^{2} C+\frac{\alpha}{1-\alpha C}(\nabla C)^{2}\right]
$$


where $C$ is the normalized ion concentration of $\mathrm{A}$ ions. $\alpha=1-D_{A} / D_{B}, D_{A}$ and $D_{B}$ are the self-diffusion coefficients for $\mathrm{A}$ ions and $\mathrm{B}$ ions respectively. The $\mathrm{A}$ and $\mathrm{B}$ ions are assumed as $\mathrm{Ag}$ and $\mathrm{Na}$ ions, respectively. In this model the parameters $D_{A}=5.1 \times 10^{-6}$ and $\alpha=0.87$ are chosen from Ref. [10]. The ion-exchange and annealing times are 20 and 35 minutes, respectively. The length of the waveguide is $4000 \mu \mathrm{m}$. The width of the waveguide on the photomask is $3 \mu \mathrm{m}$. The contour plots of the refractive index distribution for the ion-exchanged waveguide produced with a 20min ion exchange and a 35-min annealing process are presented in Fig. 2 (a). The simulated contour plots of full-vector eigenmodes of the ion-exchanged glass waveguide are presented in Fig.2 (b) and Fig.2 (c).

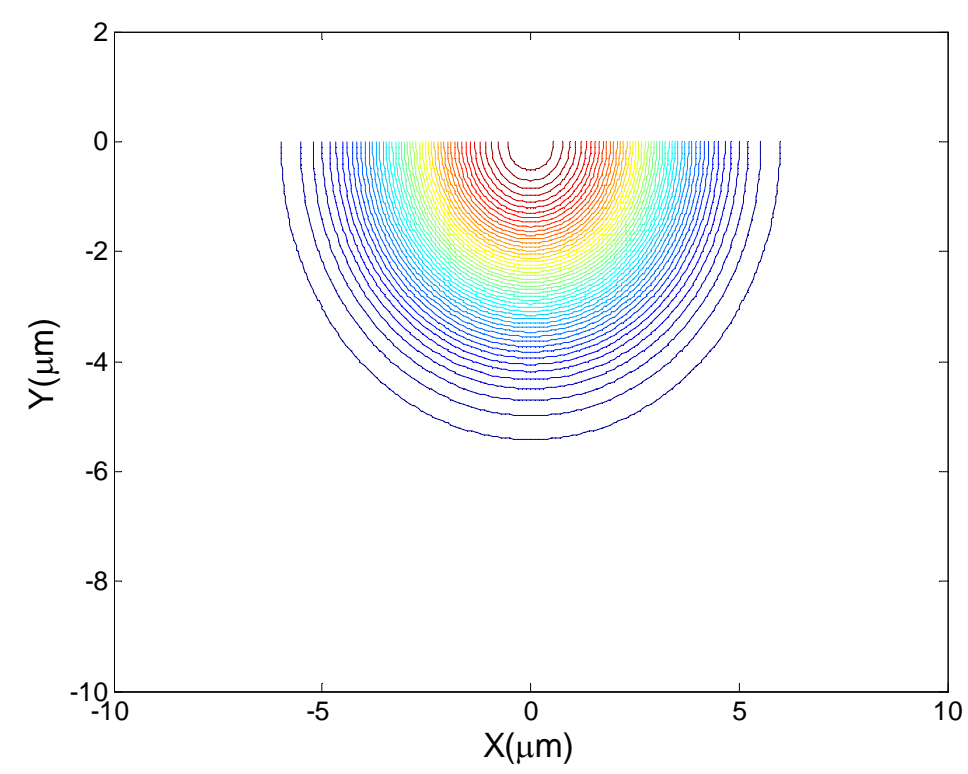

(a) 


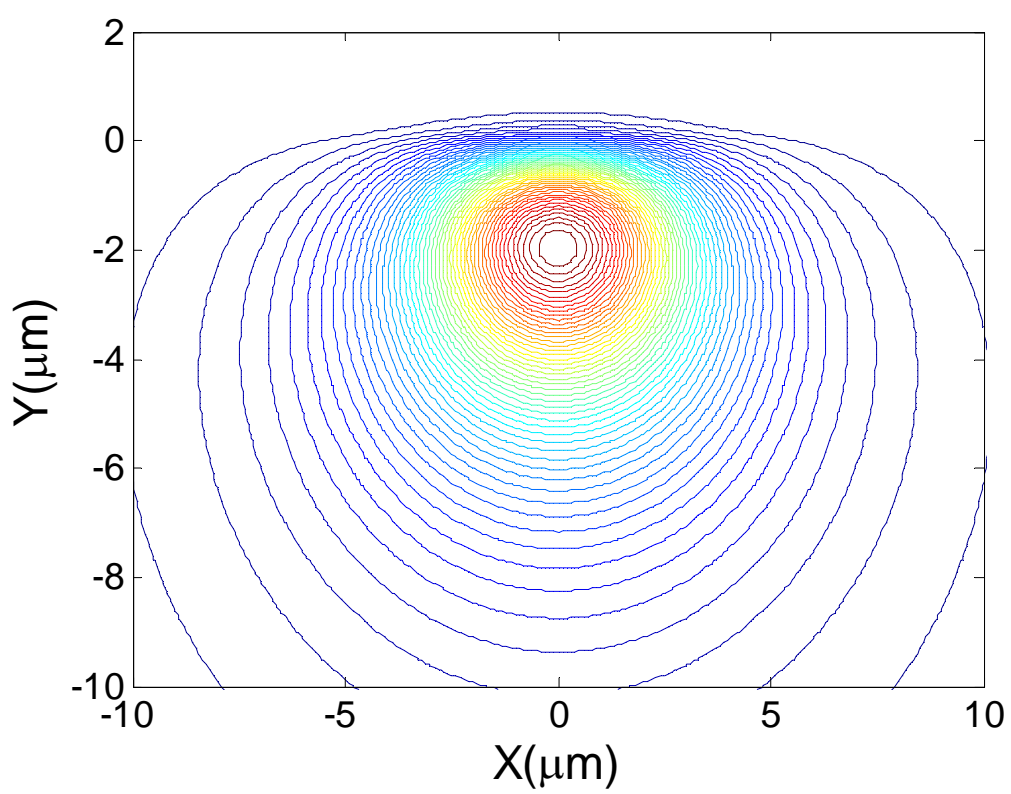

(b)

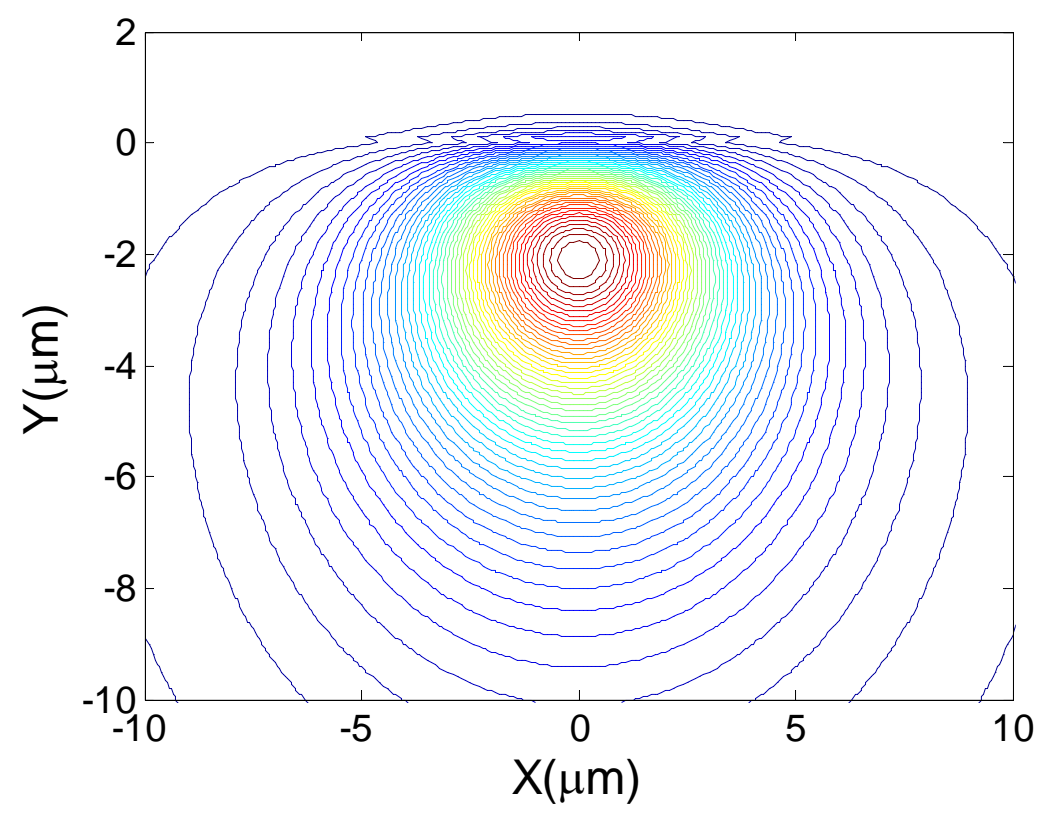

(c)

Fig. 2. (a) Refractive index profile of the ion exchanged waveguide (after an ion exchange process of 20 minutes and an annealing process of 35 minutes) and full-vector eigenmode of an ionexchanged glass waveguide: (b) $\mathrm{E}_{\mathrm{x}}$ of the quasi-TE mode; (c) $\mathrm{E}_{\mathrm{y}}$ of the quasi-TM mode.

To calculate the eigenmode of an ion exchanged waveguide with a LC overlayer, in the numerical example, the thickness of the LC layer is assumed as 10 
$\mu \mathrm{m}$ and a planar alignment (in the $\mathrm{x}$ direction) of the $\mathrm{LC}$ is chosen for the case with no external electric field (OFF state). With the 3-dimensional full-vector FD-BPM developed above, the $E_{x}$ and $E_{y}$ components for the TE and TM modes are presented in Fig. 3(a) and (b), respectively. From Fig. 3(a) one can see that a leaky mode exists in the LC layer near the waveguide, thus TE-polarized input light experiences high propagation loss in the integrated polarizer device.

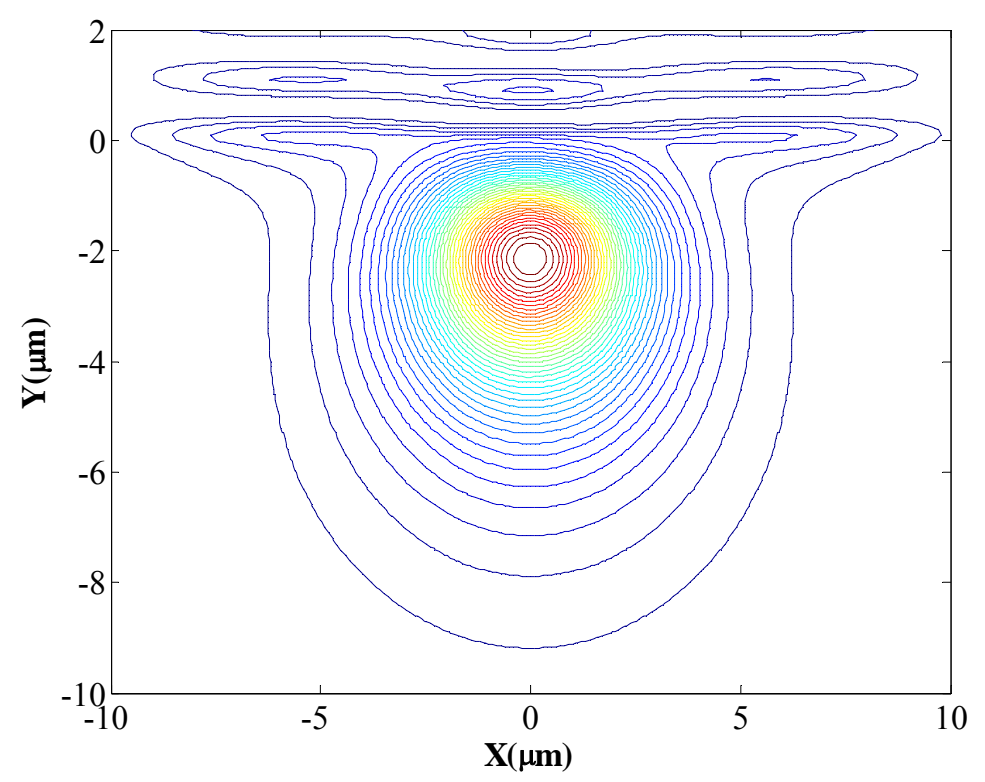

(a)

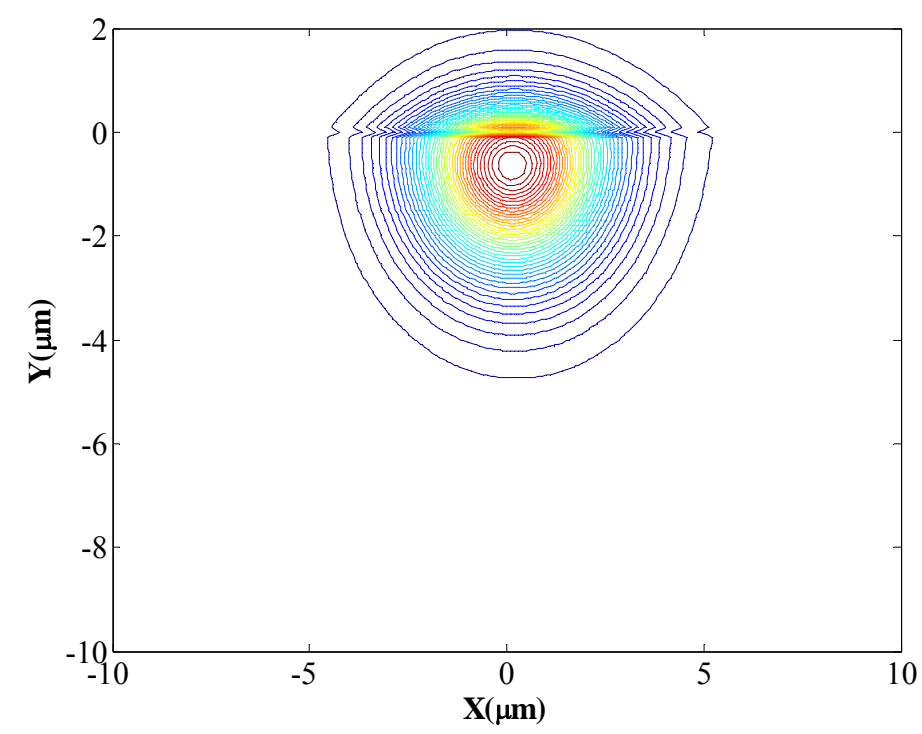

(b) 
Fig. 3. Full-vector eigenmode of an ion-exchanged glass waveguide covered by LC in the absence of electric field (OFF-state): (a) $E_{x}$ of the TE mode; (b) $E_{y}$ of the TM mode.

The transmission losses for both TE and TM modes along the propagation distance are calculated and the results of this simulation are shown in Fig. 4. From the figure one can see that in the OFF state, the propagation loss for the TE mode (E vector along the $\mathrm{x}$ axis) is higher than $-13 \mathrm{~dB}$ over the distance of $4000 \mu \mathrm{m}$ as a result of the existence of a leaky mode in the LC layer. The TM mode (E vector along the $\mathrm{y}$ axis), on the other hand, propagates in both the waveguide and the LC layer with strong guiding, and therefore the loss along the propagation in the waveguide is relatively small, circa $-5 \mathrm{~dB}$. The application of the electric field perpendicularly to the LC layer (ON-state) changes the molecular orientation of the LC so the propagation loss of the TM mode changes to $-14.42 \mathrm{~dB}$ and the transmission loss of the TE mode changes to $-5.23 \mathrm{~dB}$ over the same propagation distance of $4000 \mu \mathrm{m}$. This is caused by the changes in the propagation conditions for both TE and TM modes, as a result the TE mode is strongly guided in both the waveguide and the LC, while the TM mode experiences high loss in the waveguide as a result of the existence of a leaky mode in the LC layer. As a result the device operates as a TE passing polarizer in the $\mathrm{ON}$ state.

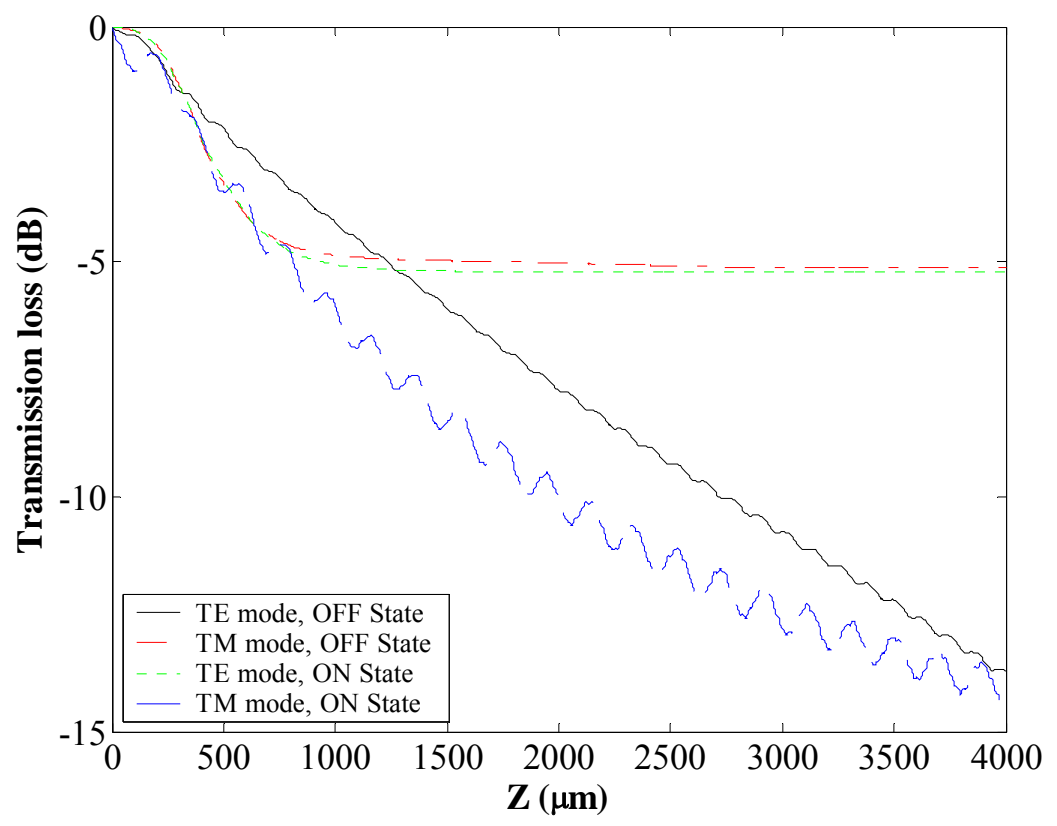

Fig. 4. Transmission losses in the optical polarizer (OFF-state) versus propagations distance, the operating wavelength is $1550 \mathrm{~nm}$. Model is solved using a 3-D beam propagation method. 
For an ion-exchanged waveguide, the pure propagation loss is caused mainly by Rayleigh scattering, which is determined by the ion concentration and the wavelength of the light. For a typical $\mathrm{Ag}^{+}-\mathrm{Na}^{+}$ion-exchanged waveguide, the theoretical propagation loss is circa $0.01 \mathrm{~dB} / \mathrm{cm}$ and the measured propagation loss can achieve $0.14 \mathrm{~dB} / \mathrm{cm} @ 1550 \mathrm{~nm}$ [14]. On the other hand, the refractive index difference between the TE and TM mode can be circa $4 \sim 5 \times 10^{-4}$ after the ion exchange process and the measured polarization dependent loss of the straight ionexchange waveguide is circa $0.4 \mathrm{~dB}$, which is very small and can be neglected in practice.

As shown in Fig. 4, the desired polarization state (both the TE mode in an ideal ON state and TM mode in an ideal OFF state) suffers a loss of circa $5 \mathrm{~dB}$ per simulation. This phenomenon is likely to be caused by the fact that deformation profiles of the LC molecules director vary for different applied voltages, further such deformation has a significant influence on the increase of the transmission loss for both TE and TM modes mainly propagating in the waveguide. For the proposed integrated polarizer component, the transmission loss from the input fiber to the output fiber can be extremely important for all operational modes with changing the voltages of the external electrodes. Hence a pure transmission loss no higher than 7 $\mathrm{dB}$ without considering the extra polarization effects caused by the applied voltages is expected for the proposed integrated polarizer device.

\section{Impact of surface anchoring}

It is known that in practice, while the LC molecules in the middle of the LC layer re-orient along the field [15], application of the electric field does not change the alignment of the LC molecules near the surface of the electrodes. In this paper strong anchoring alignment is chosen for the theoretical design.

For the chosen nematic LC (E7 from Merck), the corresponding parameters are $\left.k_{11}=11.7 \times 10^{-12} N, k_{22}=9.0 \times 10^{-12} N, k_{33}=19.5 \times 10^{-12} N, \varepsilon_{/ /}=19.2, \varepsilon_{\perp}=5.3\right)$.

With the iterative finite difference method presented in Ref. [16], the corresponding 
director profiles under the applied voltages of 5, 10 and 15 Volt are presented in Fig. 5.

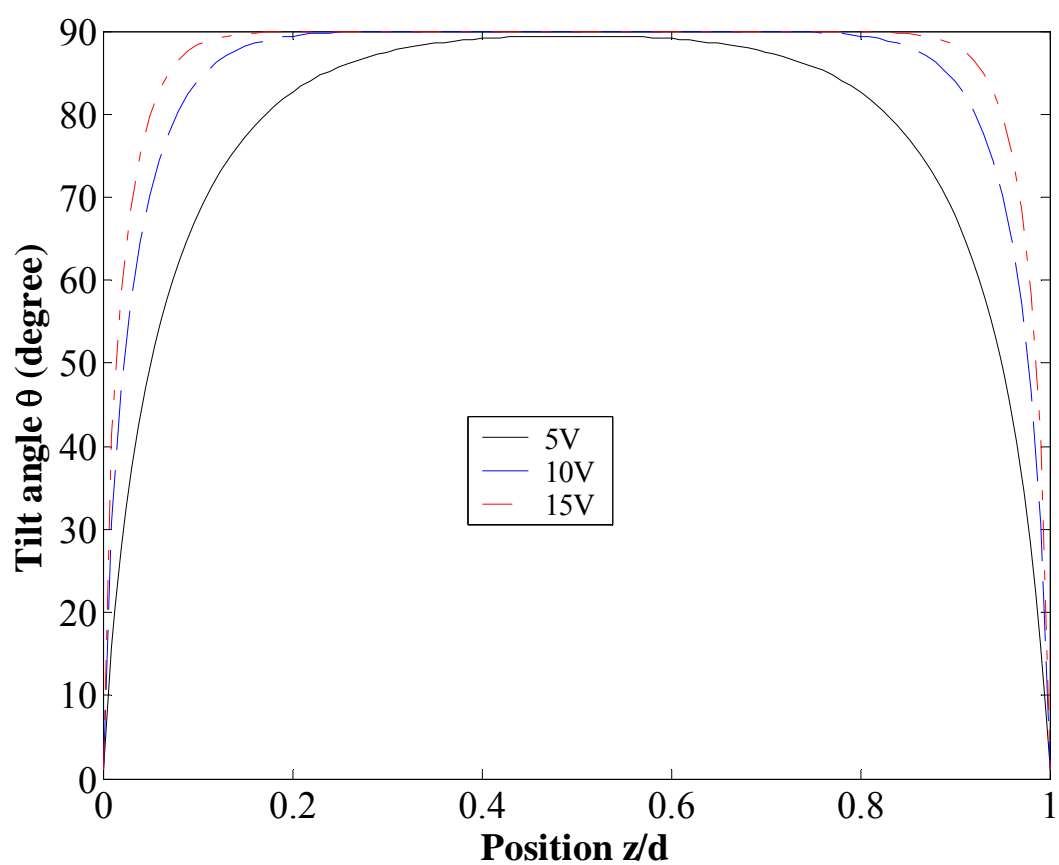

Fig. 5. The deformation profiles for an E7 LC cell under different applied voltages in the case of strong anchoring, solved by an iterative finite difference method [16].

With these profiles, the transmission losses of both TE and TM mode at different voltages in the case of strong anchoring are simulated with the model based on a 3D full-vector finite-difference beam propagation method (FV-BPM) for anisotropic materials. As shown in Fig. 6 (a)-(d), for the case of strong anchoring with applied voltages of 5, 10, 15 and $20 \mathrm{~V}$, both TE and TM losses decay as the propagation distance increases. The TM loss is higher than TE when apply the extra electric field. When the applied voltage is $5 \mathrm{~V}$, the maximum difference between the TE and TM losses are $15.23 \mathrm{~dB}$ at a propagation distance of $1758 \mu \mathrm{m}$; for the case of $10 \mathrm{~V}$, the maximum difference is $16.3 \mathrm{~dB}$ at a propagation distance of $3975 \mu \mathrm{m}$; for the case of $15 \mathrm{~V}$, the maximum difference is $14.54 \mathrm{~dB}$ at a propagation distance of $2977 \mu \mathrm{m}$; for the case of $20 \mathrm{~V}$, the maximum difference is $10 \mathrm{~dB}$ at a propagation distance of $662 \mu \mathrm{m}$. The increase in attenuation for TE loss is due to the strong interaction between the substrate surface (alignment layer) and the liquid crystal molecules near the surface of ion-exchanged waveguide. However, attenuation for 
TM modes increases also due to the mode perturbation induced by the high refractive index alignment layers. This increased value of TE to TM attenuation ratio (the extinction ratio) can be valuable in the practical design of a more efficient polarizer. The difference in simulated results between the ideal and the strong anchoring case shows that surface anchoring plays a significant role in the device performance. From Fig. 4, one can also see that significant oscillations in the value of the transmission loss over the propagation length occur for both TE and TM modes, caused by the strong interaction between the waveguide, polyimide and LC layers, when the eigenmode propagating in the ion exchange waveguide partially leaks into the thin polyimide layer with a high refractive index which results in disturbing the polarization states of both TE and TM modes. Compared with the extinction ratio of $15 \mathrm{~dB}$ for the integrated LC device reported in Ref [3], in this paper the extinction ratio of the proposed LC waveguide device with an external voltage of $11 \mathrm{~V}$ can reach a maximum value of circa $16.66 \mathrm{~dB}$, with the advantages of much simpler structure and ease of fabrication.

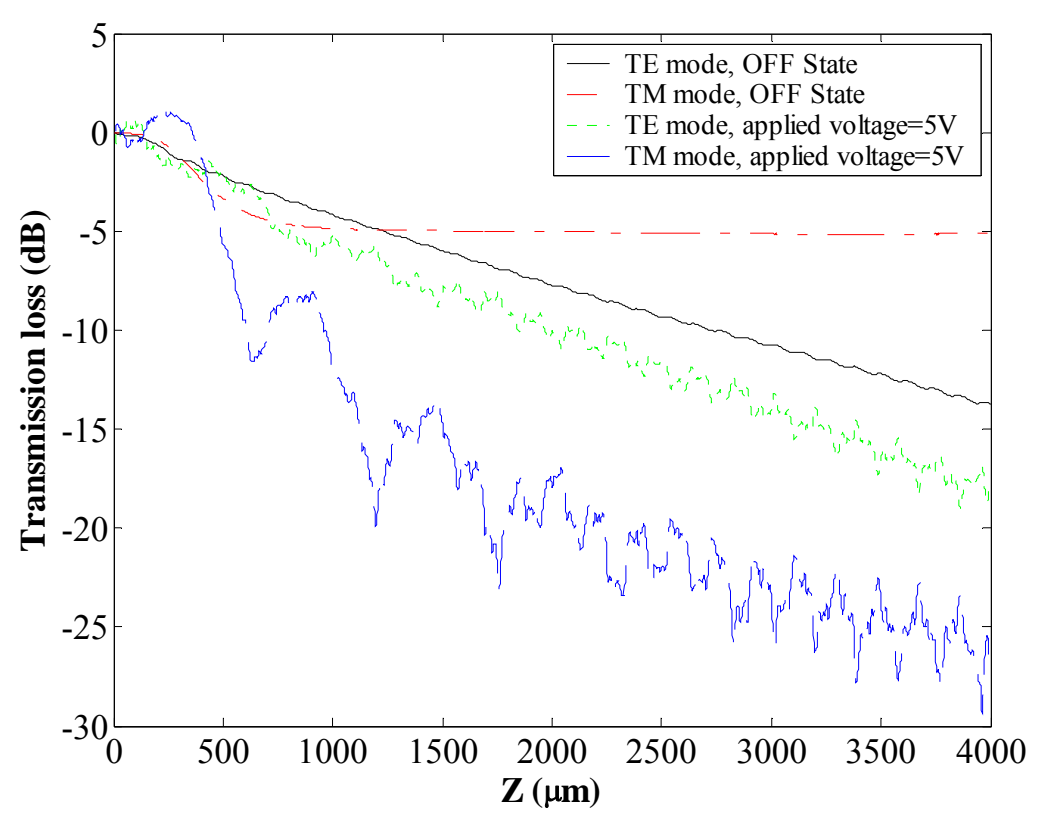

(a) 


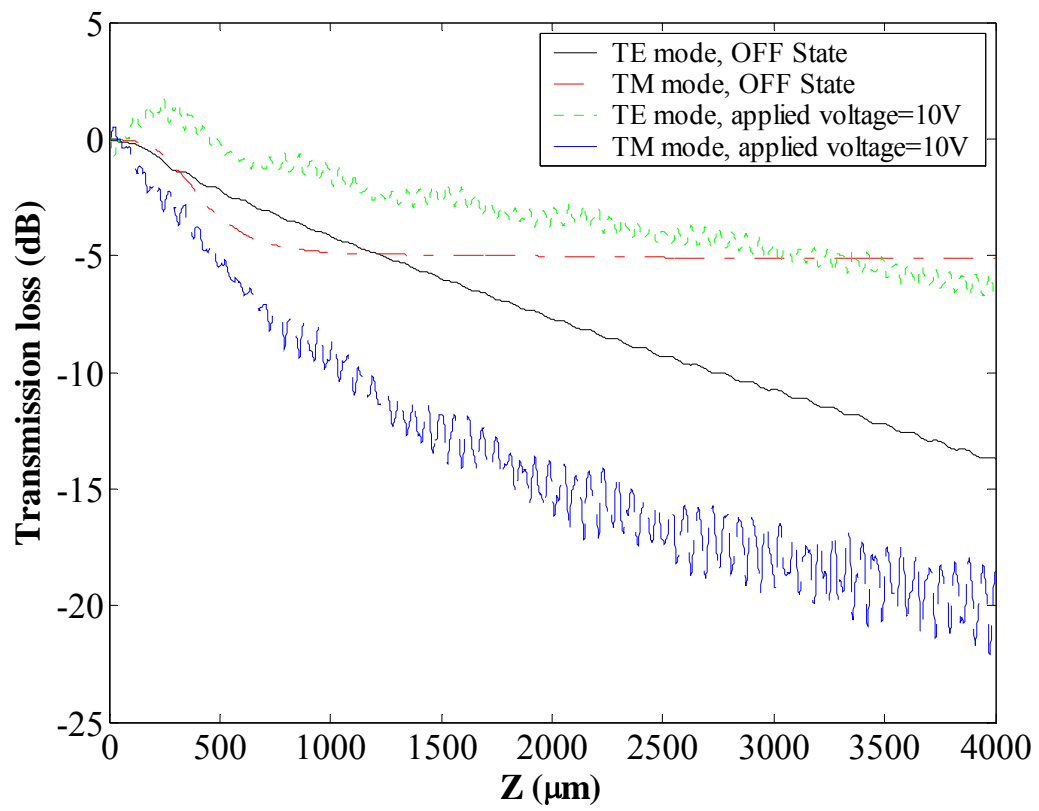

(b)

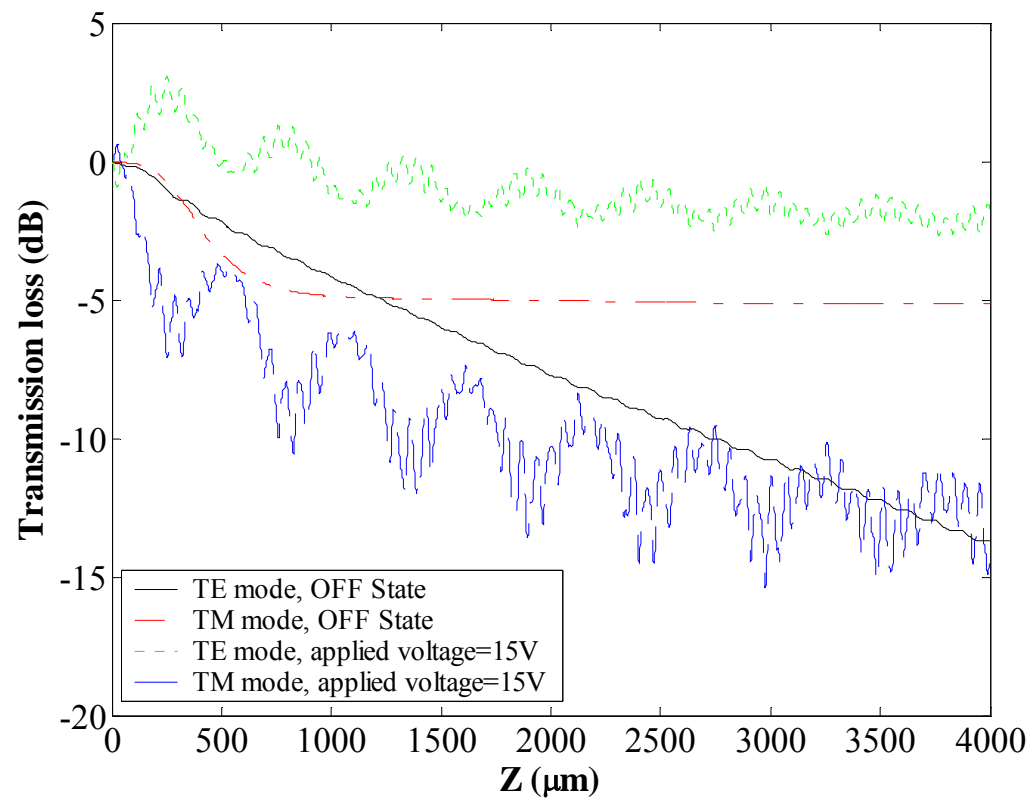

(c) 


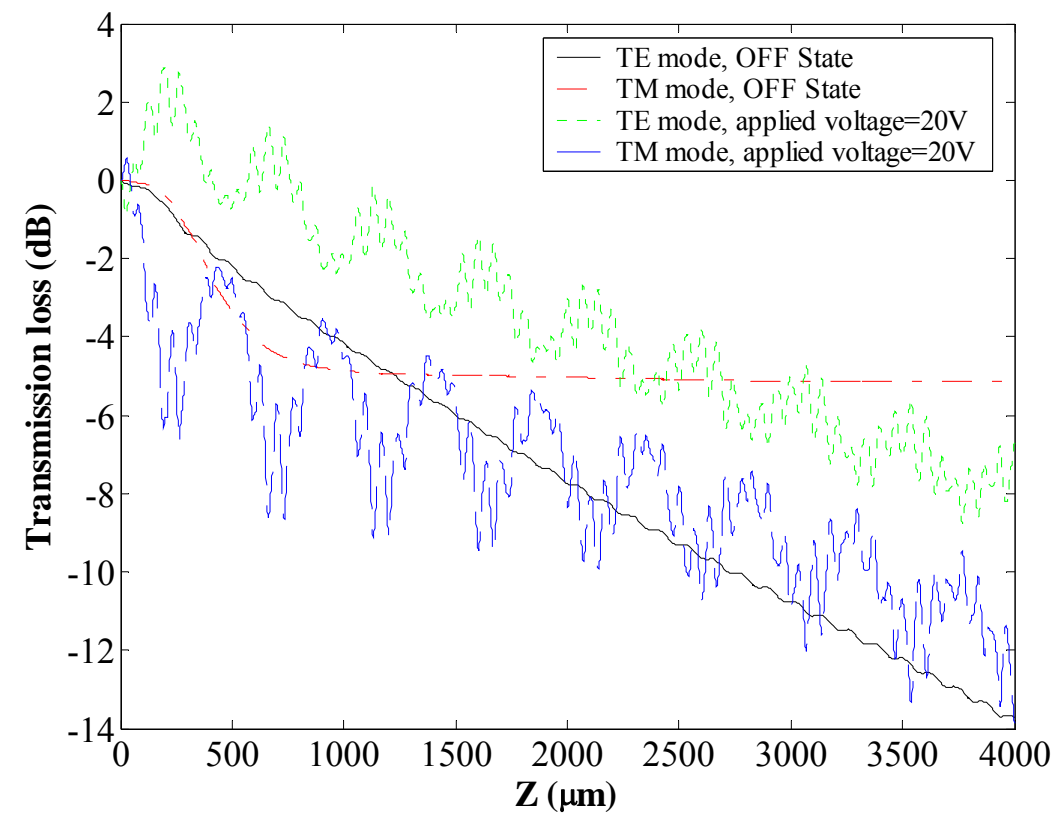

(d)

Fig. 6. Transmission loss in the optical polarizer covered by a LC layer under the influence of an electric field applied to the LC layer (in the case of strong anchoring) versus propagations distance, the applied voltage is (a) $5 \mathrm{~V}$; (b) $10 \mathrm{~V}$; (c) $15 \mathrm{~V}$; (d) $20 \mathrm{~V}$, the operating wavelength is $1550 \mathrm{~nm}$.

Models are solved using a 3-D full-vectorial FD-BPM.

To adequately characterize the TE to TM attenuation ratio value as a key factor to evaluate the performance of the proposed optical polarizer, the parameter "discrimination range" is proposed to express the difference between the transmission loss of TE and TM model at a certain propagation distance by the following definition:

$$
\text { Discrimination range }=L_{T E}-L_{T M}
$$

With this formula, the TE to TM attenuation ratio for the proposed polarizer can be characterized in a general sense. For the transmission losses of the TE and TM mode at both $\mathrm{ON}$ and OFF states presented in Fig. 6 (a) (d), the maximum discrimination ranges and the corresponding propagation distances versus the different electric voltage are presented in Fig. 7. From this figure one can see that the discrimination range increases monotonically as the voltage increases from $1 \mathrm{~V}$ to $11 \mathrm{~V}$, resulting in the performance of the proposed optical polarizer improving with an increase in magnitude of the electric field. 


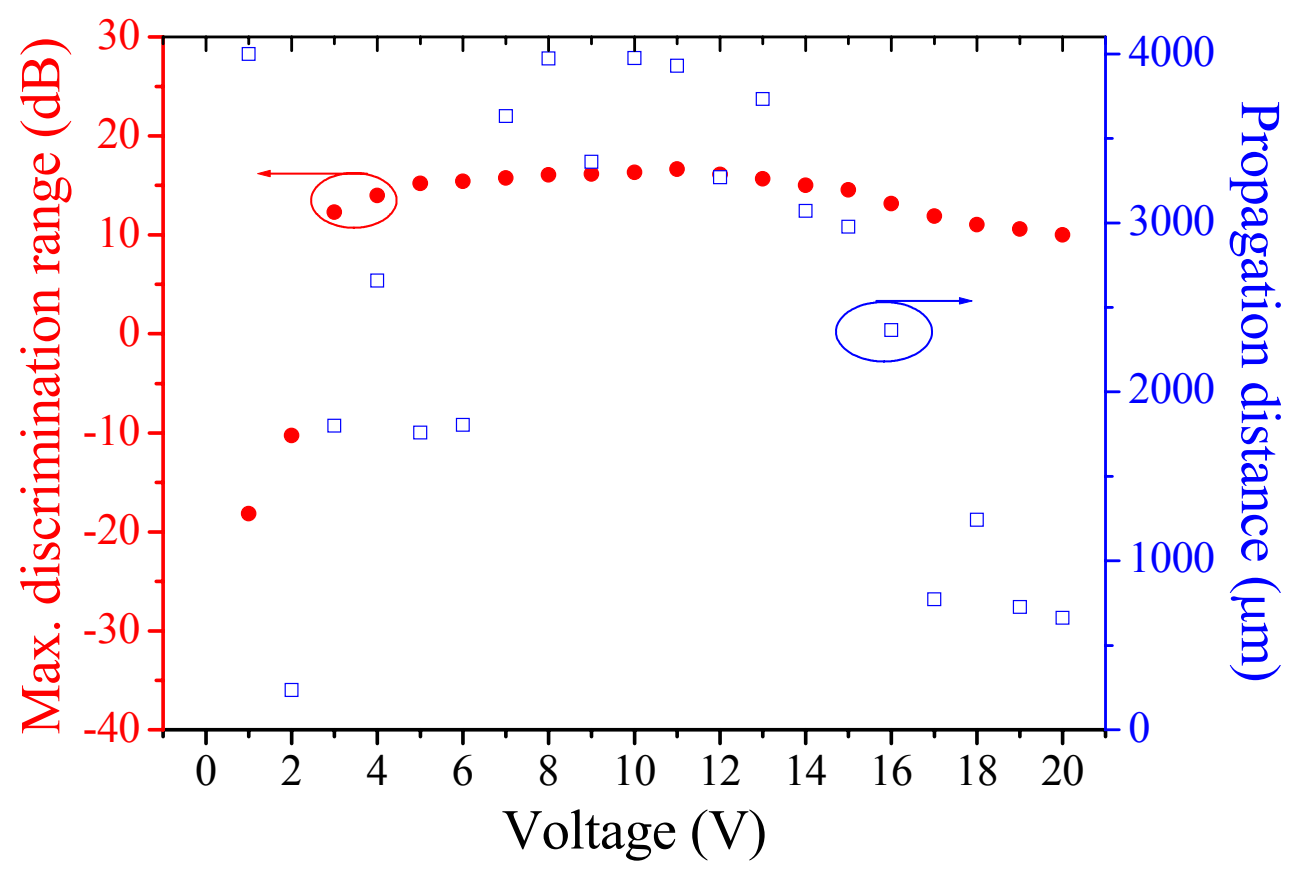

Fig. 7. The difference in transmission loss between the TE and TM modes (left Y-axis) and the corresponding propagation distance (right $\mathrm{Y}$-axis) versus the voltage of the extra electric field (in the case of strong anchoring).

\section{Conclusion}

In this paper, light propagation within an LC covered ion-exchanged glass waveguide based optical polarizer device has been investigated. The threedimensional full-vector finite difference beam propagation method for an anisotropic medium has been presented, allowing beam propagation within the waveguide polarizer to be modelled. The full vector eigenmodes of a glass waveguide with an LC covering layer have been solved, so that the beam behaviours within this device can be simulated for both ON and OFF states. The effect of strong surface anchoring on the device performance has been demonstrated numerically and the calculated results indicate that the surface anchoring of the liquid crystal is a key issue influencing the performance of the device. Although only the polarizer application is considered in this paper, the proposed integrated LC device can also be optimized and developed further as a polarization beam splitter which is widely used in optical communications or optical fibre sensing systems. Moreover, in principle, by further optimizing a number of physical parameters for the proposed LC device, such as the refractive index of the glass substrate, refractive index difference between the waveguide and 
substrate, thickness of the LC and width of the waveguide, a more compact polarizer with an improved performance can be realized. Such considerations for optimal design and experimental fabrication will be carried out in our future work.

\section{Acknowledgement}

Pengfei Wang is funded by the Irish Research Council for Science, Engineering and Technology, co-funded by the Marie-Curie Actions under FP7.

The research was partially supported by the National Natural Science Foundation of China (No. 60777038), the China-Ireland Science and Technology Collaboration Research Fund and the International cooperation project (No.20070708-3) of Jilin Provincial Science \& Technology Department of China.

\section{Reference}

[1]. N. A. Riza and S. Yuan, "Reconfigurable wavelength add-drop filtering based on a Banyan network topology and ferroelectric liquid crystal fiber-optic switches", J. Lightwave Technol.17, 1575-1584 (1999).

[2].K. Wu, J. Liu and Y. Chen, "Optical attenuator using polarization modulation and a feedback controller", US patent 5963291 (1999).

[3].K. Hirabayashi and C. Amano, "Liquid-crystal polarization controller arrays on planar waveguide circuits”, IEEE Photonics Technol. Lett. 14, 504-506 (2002).

[4]. Y. Semenova, Y. Panarin, G. Farrell and S. Dovgalets, "Liquid crystal based optical switches”, Mol. Cryst. Liq. Cryst. 413, 2521-2534 (2004).

[5].K. Nakatsuhara, T. Sasaki, H. Sato, and T. Nakagami, "Si-Waveguide with FerroElectric Liquid Crystal Cladding for Use in Optical Switching Devices”, IEICE TRANSACTIONS on Electronics, Vol. E90-C, No.5 pp.1055-1060 (2007). 
[6].Y. Xu, M. A. Uddin, P. S. Chung, and H. P. Chan, "Polymer planar waveguide device using inverted channel structure with upper liquid crystal cladding”, Optics Express, Vol. 17, No. 10, pp. 7837-7843 (2009).

[7].D. Donisi, R. Asquini, A. d'Alessandro, B. Bellini, R. Beccherelli, L. De Sio, and C. Umeton, "Integration and Characterization of LC/Polymer Gratings on Glass and Silicon Platform”, Molecular Crystals and Liquid Crystals, Vol. 516, pp. 152$158(2010)$.

[8].R. A. Betts, F. Lui and S. Dagias, "Wavelength and polarization insensitive optical splitters fabricated in $\mathrm{K}^{+} / \mathrm{Na}^{+}$Ion-exchanged glass”, IEEE Photon. Technol. Lett., Vol. 2, No. 7, pp.481-483, (1990).

[9].B. Buchold, C. Glingener, D. Culemann and E. Voges, "Polarization insensitive ion-exchanged array-waveguide grating multiplexers in glass", Fiber and Integrated Optics, Vol. 17, pp. 279-298, (1998).

[10]. B. R. West, P. Madasamy, N. Peyghambarian and S. Honkanen, "Modeling of ion-exchanged glass waveguide structures”, J. Non-Crystalline Solids, Vol. 347, pp. 18-26, (2004).

[11]. J. T. A. Carriere, J. A. Frantz, B. R. West, S. Honkanen and R. K. Kostuk, "Bend loss effects in diffused, buried waveguides", App. Opt., Vol. 44, No. 9, pp. 1698-1703, (2005).

[12]. J. M. Castro, D. F. Geraghty, B. R. West and S. Honkanen, "Fabrication and comprehensive modelling of ion-exchanged Bragg optical add-drop multiplexers", App. Opt. Vol. 43, No. 33, pp. 6166-6173, (2004).

[13]. S. Yliniemi, B. R. West and S. Honkanen, "Ion-exchanged glass waveguides with low birefringence for a broad range of waveguide widths”, App. Opt., Vol. 44, No. 16, pp. 3358-3363, (2005). 
[14]. Z. He, Y. Li, Y. Li, Y. Zhang, L. Liu, and L. Xu, "Low-loss channel waveguides and Y-splitter formed by ion-exchange in silica-on-silicon", Optics Express, Vol. 16, No. 5, pp. 3172-3177, (2008).

[15]. M. P. Allen, "Molecular simulation and theory of liquid crystal surface anchoring”, Molecular Physics, Vol. 96, No. 9, pp. 1391-1397, (1999).

[16]. Q. Wang, S. He, F. Yu and N. Huang, "Iterative finite-difference method for calculating the distribution of a liquid-crystal director”, Opt. Eng., Vol. 40, No. 11, pp. 2552-2557, (2001). 